\title{
GLASGOW UNIVERSITY RADIOCARBON MEASUREMENTS II
}

\author{
M. ERGIN, D. D. HARKNESS, and A. WALTON
}

Chemistry Department, The University, Glasgow, W.2

A third radiocarbon counting system has been established in the Chemistry Department, University of Glasgow, since April, 1968. Operating conditions for the previous systems have remained essentially as described by Baxter et al. (1969).

The counting assembly was supplied by Johnston Laboratories, Inc., Baltimore and consists of 2.6L internal gas counter and a concentric multiple anode anticoincidence meson detector. The counters are encased in a 4-in.-thick shield manufactured from aged lead by J. Girdler and Co., London.

$\mathrm{CO}_{2}$ is employed as the counting gas at a constant filling pressure of $760 \mathrm{~mm} . \mathrm{Hg}$ at $15^{\circ} \mathrm{C}$. Operational parameters are as follows: (1) anticoincidence plateau: greater than $800 \mathrm{v}$ long with slope less than $0.5 \%$ per $100 \mathrm{v}$; (2) detector operating voltage: $3.48 \pm 0.05 \mathrm{Kv}$. Adjustment is made within this range to ensure identical gas gain for all gases counted; (3) detector background count rate: $5.53 \pm 0.12( \pm 2 \sigma)$ counts/ min. at 1013 mbar. A linear variation of background count rate with barometric pressure, amounts to -0.01 count $/ \mathrm{min}$./mbar; (4) net activity of NBS oxalic acid modern standard: $14.37 \pm 0.08( \pm 2 \sigma)$ counts/min., after correction for fractionation and decay.

$\mathrm{CO}_{2}$ samples are normally stored for 14 days prior to counting to allow for radon decay. The presence of radon, however, is monitored via energy discrimination during each counting sequence. When necessary, a correction is applied to the total count rate to allow for the contribution of radon and its beta active daughter products.

Samples are counted at least twice and several days apart to give a minimum total of 60,000 counts. Modern standard and background activities are monitored weekly to check counter performance.

Mass spectrometric analysis for fractionation correction have been performed at The National Physical Laboratory, Teddington.

Calculations are based on the Lamont VIII formulae (Radiocarbon 1961, v. 3, p. 176-204) and errors arising from uncertainties in $\mathrm{C}^{14}$ measurement are quoted to one standard deviation $\left(1_{\sigma}\right)$.

\section{ACKNOWLEDGMENTS}

Financial support for different aspects of this research has been provided by the Medical Research Council and the Natural Environment Research Council. Our gratitude is again extended to those persons and organizations throughout the world who have assisted us in the supply of suitable samples. The National Physical Laboratory has again cooperated in providing facilities for $\mathrm{C}^{13} / \mathrm{C}^{12}$ measurement. Mrs. $\mathrm{M}$. Currie provided excellent technical assistance in the laboratory. 


\section{SAMPLE DESCRIPTIONS}

\section{INTERCALIBRATION SAMPLES}

Prior to routine measurement of $\mathrm{C}^{14}$ activities with the new system intercalibration was performed in conjunction with the established radiocarbon counting facilities at Glasgow (Baxter et al., 1969).

\section{GU-67. Kilphedir hut circles, Sutherland, Scotland A.D. 28}

Charcoal. Comment: sample previously described and reported under GU-10, $1908 \pm 60$, GU-11, $2064 \pm 55$ and L-1061, $2100 \pm 80$.

\section{Snowdon, Wales 1968}

Atmospheric $\mathrm{CO}_{2}$ samples, counted as $\mathrm{CO}_{2}$ and then converted to $\mathrm{CH}_{4}$ for measurement on alternative counting systems. Coll. by Central Electricity Generating Board at Cwm Dyli, Mt. Snowdon, Wales, alt $300 \mathrm{ft}\left(53^{\circ} 03^{\prime} \mathrm{N} \mathrm{Lat}, 04^{\circ} 00^{\prime} \mathrm{W}\right.$ Long).

GU-83.

\begin{tabular}{ccc}
$\delta \mathrm{C}^{14} \%$ & $\delta \mathrm{C}^{13 \%} \%$ & $\Delta \%$ \\
\hline $60.0 \pm 0.9$ & -21.3 & $58.9 \pm 0.9$
\end{tabular}

$\mathrm{CO}_{2}$ coll. April 1968. Counted as $\mathrm{CO}_{2}$ on new system.

$$
\begin{aligned}
& 59.8 \pm 0.7 \quad-21.3 \\
& \text { Counted as } \mathrm{CH}_{4} \text { on system } 1 .
\end{aligned}
$$

$\mathrm{CO}_{2}$ coll. April 1968. Counted as $\mathrm{CH}_{4}$ on system 1 .

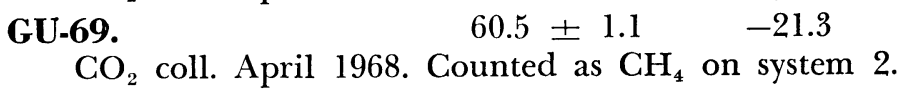

GU-70.
$\mathrm{CO}_{2}$ coll. June 1968. Counted as $\mathrm{CO}_{2}$ on new system.

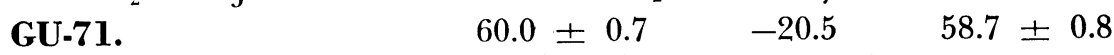

$\mathrm{CO}_{2}$ coll. June 1968. Counted as $\mathrm{CH}_{4}$ on system 1.

$58.7 \pm 0.8$

$59.4 \pm 1.2$

Agreement between systems is satisfactory; no further designation of counting system is deemed necessary.

\section{ATMOSPHERIC $\mathrm{CO}_{2}$ SAMPLES}

\section{A. Ground level}

Data reported here are derived from atmospheric $\mathrm{CO}_{2}$ samples coll. at various sites in the U.K. and throughout the world. Measurements were made as part of 2 continuing research programs, viz., (a) $\mathrm{C}^{\mathbf{1 4}}$ concentrations in humans in relationship to those of their immediate environment (Harkness and Walton, 1969) and (b) transport of $\mathrm{C}^{14}$ within the "dynamic" carbon reservoir (Walton et al., 1969).

$\mathrm{CO}_{2}$ coll. by exposure of carbonate free $8 \mathrm{~N} \mathrm{KOH}$ solution to atmosphere for each calendar month.

\section{Snowdon series}

$\mathrm{CO}_{2}$ coll. by the Central Electricity Generating Board in a ventilated cabinet at Cwm Dyli Power Sta. on E slope of Mt. Snowdon $\left(53^{\circ} 03^{\prime}\right.$ $\mathrm{N}$ Lat, $04^{\circ} 00^{\prime} \mathrm{W}$ Long). 
Snowdon series, 1967

\begin{tabular}{clccc}
$\begin{array}{c}\text { Sample } \\
\text { no. }\end{array}$ & $\begin{array}{l}\text { Coll. } \\
\text { date }\end{array}$ & $\delta \mathrm{C}^{14} \%$ & $\delta \mathrm{C}^{13 \%} \%$ & $\Delta \%$ \\
\hline GU-72 & May & $64.4 \pm 0.8$ & -19.9 & $62.7 \pm 0.8$ \\
GU-73 & June & $54.0 \pm 0.9$ & -18.1 & $51.8 \pm 1.0$ \\
GU-74 & July & $64.8 \pm 1.5$ & -17.4 & $62.2 \pm 1.6$ \\
GU-75 & Aug. & $60.0 \pm 0.6$ & -22.8 & $59.3 \pm 0.7$ \\
GU-76 & Sept. & $62.1 \pm 0.8$ & -17.8 & $59.8 \pm 0.8$ \\
GU-77 & Oct. & $59.8 \pm 0.6$ & -18.0 & $57.6 \pm 0.7$ \\
GU-78 & Nov. & $59.1 \pm 0.8$ & -20.2 & $57.6 \pm 0.8$ \\
GU-79 & Dec. & $54.3 \pm 1.4$ & -18.3 & $52.2 \pm 1.5$
\end{tabular}

Snowdon series, 1968

$\begin{array}{lllll}\text { GU-80 } & \text { Jan. } & 58.5 \pm 1.4 & -21.4 & 57.3 \pm 1.5 \\ \text { GU-81 } & \text { Feb. } & 52.9 \pm 1.3 & -21.6 & 51.9 \pm 1.4 \\ \text { GU-82 } & \text { March } & 57.1 \pm 1.4 & -20.7 & 55.8 \pm 1.5 \\ \text { GU-83 } & \text { April } & 60.0 \pm 0.9 & -21.3 & 58.9 \pm 0.9 \\ \text { GU-84 } & \text { May } & 60.3 \pm 0.8 & -20.5 & 58.8 \pm 0.9 \\ \text { GU-85 } & \text { June } & 61.6 \pm 0.8 & -20.5 & 60.2 \pm 1.0 \\ \text { GU-86 } & \text { July } & 61.9 \pm 0.9 & -20.6 & 60.5 \pm 1.0 \\ \text { GU-87 } & \text { Aug. } & 56.0 \pm 1.0 & -20.0 & 54.4 \pm 1.1 \\ \text { GU-88 } & \text { Sept. } & 59.3 \pm 1.0 & -20.4 & 57.8 \pm 1.1 \\ \text { GU-89 } & \text { Oct. } & 55.8 \pm 1.4 & -19.9 & 54.9 \pm 1.4 \\ \text { GU-90 } & \text { Nov. } & 54.9 \pm 1.2 & -21.5 & 54.0 \pm 1.3 \\ \text { GU-91 } & \text { Dec. } & 50.7 \pm 0.9 & -22.9 & 50.0 \pm 1.0\end{array}$

\section{Snowdon series, 1969}

$\begin{array}{lllll}\text { GU-92 } & \text { Jan. } & 57.5 \pm 1.3 & -21.6 & 56.5 \pm 1.4 \\ \text { GU-93 } & \text { Feb. } & 55.1 \pm 0.8 & -24.5 & 55.0 \pm 0.9 \\ \text { GU-95 } & \text { April } & 57.4 \pm 0.7 & -21.2 & 56.2 \pm 0.8 \\ \text { GU-96 } & \text { May } & 57.9 \pm 0.8 & -20.3 & 56.4 \pm 0.9 \\ \text { GU-97 } & \text { June } & 52.4 \pm 0.8 & -19.2 & 50.7 \pm 0.9 \\ \text { GU-98 } & \text { July } & 56.8 \pm 0.8 & -21.4 & 55.7 \pm 0.8 \\ \text { GU-99 } & \text { Aug. } & 54.9 \pm 0.8 & -19.5 & 53.2 \pm 0.9\end{array}$

Comment: sampling station is remote from any source of fossil fuel $\mathrm{CO}_{2}$ or possible contamination by $\mathrm{C}^{14} \mathrm{O}_{2}$ from nuclear establishments. A seasonal variation in the tropospheric $\mathrm{C}^{14}$ concentration is evident, and is in agreement with present theories of stratospheric/tropospheric mixing patterns.

\section{Chilton, England series}

$\mathrm{CO}_{2}$ coll. by the United Kingdom Atomic Energy Comm. at a site adjacent to A.E.R.E. Harwell (51 $31^{\prime} \mathrm{N}$ Lat, $01^{\circ} 20^{\prime} \mathrm{W}$ Long). 


\section{Chilton series, 1967}

\begin{tabular}{clccc}
$\begin{array}{c}\text { Sample } \\
\text { no. }\end{array}$ & $\begin{array}{l}\text { Coll. } \\
\text { date }\end{array}$ & $\delta \mathrm{C}^{14 \%} \%$ & $\delta \mathrm{C}^{13 \%} \%$ & $\Delta \%$ \\
\hline GU-100 & May & $78.2 \pm 0.9$ & -24.0 & $77.2 \pm 1.0$ \\
GU-101 & June & $70.8 \pm 0.8$ & -22.1 & $69.8 \pm 0.9$ \\
GU-102 & July & $67.3 \pm 0.4$ & -20.3 & $65.8 \pm 0.6$ \\
GU-103 & Aug. & $60.3 \pm 1.6$ & -24.7 & $59.3 \pm 1.7$ \\
GU-104 & Sept. & $68.0 \pm 0.8$ & -21.1 & $66.8 \pm 0.8$ \\
GU-105 & Oct. & $61.0 \pm 0.8$ & -25.3 & $61.1 \pm 1.0$ \\
GU-106 & Nov. & $58.2 \pm 1.0$ & -22.9 & $57.5 \pm 1.2$ \\
GU-107 & Dec. & $62.8 \pm 1.2$ & -22.5 & $61.8 \pm 1.2$
\end{tabular}

\section{Chilton series, 1968}

$\begin{array}{lllll}\text { GU-108 } & \text { Jan. } & 54.8 \pm 1.4 & -25.1 & 54.9 \pm 1.4 \\ \text { GU-109 } & \text { Feb. } & 49.6 \pm 0.5 & -24.9 & 48.7 \pm 0.6 \\ \text { GU-110 } & \text { March } & 57.3 \pm 1.0 & -22.0 & 56.3 \pm 1.0 \\ \text { GU-111 } & \text { April } & 62.5 \pm 0.9 & -25.5 & 62.6 \pm 1.0 \\ \text { GU-112 } & \text { May } & 63.0 \pm 0.9 & -21.3 & 61.8 \pm 1.0 \\ \text { GU-113 } & \text { June } & 63.5 \pm 0.9 & -21.0 & 62.2 \pm 1.0 \\ \text { GU-114 } & \text { July } & 61.0 \pm 1.3 & -24.6 & 60.9 \pm 1.4 \\ \text { GU-115 } & \text { Aug. } & 60.3 \pm 1.3 & -23.4 & 59.7 \pm 1.4 \\ \text { GU-116 } & \text { Sept. } & 63.4 \pm 1.1 & -24.1 & 63.1 \pm 1.2 \\ \text { GU-117 } & \text { Oct. } & 79.9 \pm 1.4 & -25.6 & 80.1 \pm 1.5 \\ \text { GU-118 } & \text { Nov. } & 52.3 \pm 1.2 & -26.4 & 52.8 \pm 1.3 \\ \text { GU-119 } & \text { Dec. } & 49.2 \pm 1.1 & -22.4 & 48.5 \pm 1.2\end{array}$

Chilton series, 1969

$\begin{array}{lllll}\text { GU-120 } & \text { Jan. } & 65.7 \pm 0.8 & -25.6 & 65.9 \pm 0.9 \\ \text { GU-121 } & \text { Feb. } & 55.1 \pm 0.8 & -29.4 & 56.5 \pm 0.9 \\ \text { GU-122 } & \text { March } & 55.1 \pm 0.9 & -25.5 & 55.3 \pm 1.0 \\ \text { GU-123 } & \text { April } & 64.8 \pm 0.8 & -24.7 & 64.7 \pm 0.8 \\ \text { GU-124 } & \text { May } & 56.9 \pm 0.7 & -24.5 & 56.8 \pm 0.7 \\ \text { GU-125 } & \text { June } & 73.6 \pm 0.8 & -22.8 & 72.6 \pm 0.9 \\ \text { GU-126 } & \text { July } & 59.2 \pm 0.8 & -22.9 & 58.5 \pm 0.9 \\ \text { GU-127 } & \text { Aug. } & 71.0 \pm 0.8 & -23.6 & 70.6 \pm 0.9\end{array}$

Comment: occasional high $\mathrm{C}^{14}$ concentrations would appear to indicate localized atmospheric contamination from adjacent nuclear establishment (ca. $2 \mathrm{~km}$ away). A study of the above data relative to prevailing wind direction at sampling site is being made to clarify this possibility.

\section{Lerwick, Scotland series}

Samples coll. by Meteorologic Office in their ventilated East hut, Lerwick $\left(60^{\circ} 08^{\prime} \mathrm{N}\right.$ Lat, $01^{\circ} 11^{\prime} \mathrm{W}$ Long). 
Lerwick series, 1967

\begin{tabular}{lcccc}
$\begin{array}{l}\text { Sample } \\
\text { no. }\end{array}$ & $\begin{array}{l}\text { Coll. } \\
\text { date }\end{array}$ & $\delta \mathrm{C}^{14 \%}$ & $\delta \mathrm{C}^{13 \%} \%$ & $\Delta \%$ \\
\hline GU-128 & Nov. & $65.0 \pm 1.1$ & -22.6 & $64.2 \pm 1.2$ \\
Lerwick series, $\mathbf{1 9 6 8}$ & & & \\
GU-129 & Jan. & $62.4 \pm 1.2$ & -21.2 & $61.1 \pm 1.2$ \\
GU-130 & April & $68.1 \pm 1.2$ & -22.7 & $67.3 \pm 1.3$ \\
GU-131 & July & $64.0 \pm 0.9$ & -18.4 & $61.8 \pm 1.0$ \\
GU-132 & Oct. & $58.8 \pm 1.0$ & -19.9 & $57.2 \pm 1.0$
\end{tabular}

Victoria, B.C. series

Samples coll. by Defence Research Establishment Pacific, Canada, in covered box with gauze sides to allow free circulation of air $\left(48^{\circ} 25^{\prime}\right.$ N Lat, $123^{\circ} 19^{\prime}$ W Long).

Victoria series, 1967

\begin{tabular}{llcrc}
$\begin{array}{c}\text { Sample } \\
\text { no. }\end{array}$ & $\begin{array}{c}\text { Coll. } \\
\text { date }\end{array}$ & $\delta \mathrm{C}^{14} \%$ & $\delta \mathrm{C}^{13 \%}$ & $\Delta \%$ \\
\hline GU-133 & Jan. & $60.4 \pm 0.8$ & -18.4 & $58.3 \pm 0.9$ \\
GU-134 & April & $63.9 \pm 0.9$ & -17.6 & $61.5 \pm 0.9$ \\
GU-135 & July & $65.1 \pm 0.9$ & -18.3 & $62.9 \pm 1.0$ \\
GU-136 & Oct. & $59.4 \pm 0.8$ & -18.0 & $57.1 \pm 0.9$ \\
\multicolumn{2}{l}{ Victoria series, $\mathbf{1 9 6 8}$} & & & \\
GU-137 & Jan. & $58.4 \pm 0.9$ & -20.7 & $57.0 \pm 1.0$ \\
GU-138 & April & $68.4 \pm 1.0$ & -21.9 & $67.4 \pm 1.1$ \\
GU-139 & May & $66.0 \pm 1.0$ & -20.2 & $64.4 \pm 1.1$ \\
GU-140 & Sept. & $53.5 \pm 0.9$ & -21.8 & $52.6 \pm 1.0$ \\
GU-141 & Dec. & $53.0 \pm 1.0$ & -21.5 & $51.9 \pm 1.0$
\end{tabular}

\section{Gibraltar series}

Samples coll. by Meteorologic Office, R.A.F. Gilbraltar, in wellventilated room, adjacent to open window $\left(36^{\circ} 09^{\prime} \mathrm{N}\right.$ Lat, $05^{\circ} 21^{\prime} \mathrm{W}$ Long).

\section{Gibraltar series, 1967}

\begin{tabular}{llccc}
$\begin{array}{c}\text { Sample } \\
\text { no. }\end{array}$ & $\begin{array}{l}\text { Coll. } \\
\text { date }\end{array}$ & $\delta \mathrm{C}^{14 \%}$ & $\delta \mathrm{C}^{13 \%} \%$ & $\Delta \%$ \\
\hline GU-142 & Sept. & $64.9 \pm 1.1$ & -19.0 & $62.9 \pm 1.2$ \\
GU-143 & Nov. & $69.1 \pm 1.6$ & -21.6 & $68.0 \pm 1.7$
\end{tabular}

Gibraltar series, 1968

$\begin{array}{lllll}\text { GU-144 } & \text { Jan. } & 67.8 \pm 0.8 & -20.1 & 66.2 \pm 0.9 \\ \text { GU-145 } & \text { April } & 57.4 \pm 1.1 & -20.4 & 56.0 \pm 1.2 \\ \text { GU-146 } & \text { July } & 52.6 \pm 0.9 & -23.2 & 52.0 \pm 1.0 \\ \text { GU-147 } & \text { Oct. } & 67.0 \pm 1.1 & -22.3 & 66.1 \pm 1.2 \\ \text { GU-148 } & \text { Nov. } & 54.0 \pm 1.2 & -21.2 & 52.9 \pm 1.3\end{array}$




\section{Hong Kong series}

Samples coll. by Meteorologic Office at Tates Cairn radar sta. in Stevenson screen which shelters samples from both rain and dry deposition $\left(22^{\circ} 18^{\prime} \mathrm{N}\right.$ Lat, $14^{\circ} 10^{\prime} \mathrm{E}$ Long).

\begin{tabular}{llccc}
$\begin{array}{l}\text { Hong Kong series, } 1967 \\
\text { Sample } \\
\text { no. }\end{array}$ & $\begin{array}{l}\text { Coll. } \\
\text { date }\end{array}$ & $\delta \mathrm{C}^{14} \%$ & $\delta \mathrm{C}^{13 \%} \%$ & $\Delta \%$ \\
\hline GU-149 & Jan. & $60.4 \pm 0.9$ & -26.3 & $60.8 \pm 1.0$ \\
GU-150 & April & $61.2 \pm 0.9$ & -25.5 & $61.3 \pm 0.9$ \\
GU-151 & July & $55.2 \pm 0.8$ & -26.2 & $55.6 \pm 0.9$ \\
GU-152 & Nov. & $51.1 \pm 1.0$ & -27.0 & $51.7 \pm 1.1$ \\
\multicolumn{1}{l}{ Hong Kong } & series, 1968 & & & \\
GU-153 & Jan. & $54.0 \pm 1.1$ & -28.1 & $55.0 \pm 1.2$ \\
GU-154 & April & $56.6 \pm 1.0$ & -26.2 & $56.9 \pm 1.1$ \\
GU-155 & July & $52.6 \pm 1.1$ & -21.4 & $51.5 \pm 1.2$ \\
GU-156 & Nov. & $54.7 \pm 1.0$ & -24.4 & $54.5 \pm 1.0$
\end{tabular}

\section{Singapore series}

Samples coll. by Meteorologic Office, R.A.F. Changi, Singapore, at airport ( $01^{\circ} 22^{\prime} \mathrm{N}$ Lat, $103^{\circ} 59^{\prime} \mathrm{E}$ Long).

\section{Singapore series, 1968}

\begin{tabular}{llccc}
$\begin{array}{c}\text { Sample } \\
\text { no. }\end{array}$ & $\begin{array}{l}\text { Coll. } \\
\text { date }\end{array}$ & $\delta \mathrm{C}^{14 \%}$ & $\delta \mathrm{C}^{13 \%} \%$ & $\Delta \%$ \\
\hline GU-157 & Jan. & $59.0 \pm 0.8$ & -22.0 & $58.1 \pm 0.9$ \\
GU-158 & April & $57.4 \pm 0.8$ & -23.8 & $57.0 \pm 1.0$ \\
GU-159 & July & $53.2 \pm 1.1$ & -25.5 & $53.4 \pm 1.2$ \\
GU-160 & Oct. & $51.1 \pm 1.1$ & -23.6 & $50.7 \pm 1.2$
\end{tabular}

\section{Suva, Fiji Island series}

Samples coll. by Meteorologic Office in instrument hut $\left(18^{\circ} 09^{\prime}\right.$ S Lat, $178^{\circ} 27^{\prime}$ E Long).

\section{Fiji Island series, 1967}

\begin{tabular}{llccc}
$\begin{array}{c}\text { Sample } \\
\text { no. }\end{array}$ & $\begin{array}{l}\text { Coll. } \\
\text { date }\end{array}$ & $\delta \mathrm{C}^{14 \%} \%$ & $\delta \mathrm{C}^{13 \%} \%$ & $\Delta \%$ \\
\hline GU-161 & Jan. & $61.8 \pm 0.8$ & -18.5 & $59.7 \pm 0.9$ \\
GU-162 & April & $58.1 \pm 0.8$ & -18.9 & $56.2 \pm 0.9$ \\
GU-163 & July & $58.5 \pm 0.9$ & -18.1 & $56.3 \pm 1.0$ \\
GU-164 & Oct. & $64.5 \pm 1.2$ & -18.0 & $62.2 \pm 1.3$
\end{tabular}


Fiji Island series, 1968

$\begin{array}{lllll}\text { GU-165 } & \text { Jan. } & 54.9 \pm 1.1 & -20.8 & 53.6 \pm 1.2 \\ \text { GU-166 } & \text { April } & 55.4 \pm 0.9 & -21.2 & 54.3 \pm 1.0 \\ \text { GU-167 } & \text { July } & 56.1 \pm 0.9 & -21.9 & 55.2 \pm 1.0 \\ \text { GU-168 } & \text { Oct. } & 54.6 \pm 1.0 & -21.7 & 53.5 \pm 1.0\end{array}$

\section{Pretoria series}

Samples coll. by Atomic Energy Board, Pelindaba, Pretoria, in Stephenson screen housing a variety of meteorologic instruments $\left(25^{\circ}\right.$ $45^{\prime}$ S Lat, $28^{\circ} 16^{\prime}$ E Long).

\section{Pretoria series, 1968}

\begin{tabular}{llccc}
$\begin{array}{c}\text { Sample } \\
\text { no. }\end{array}$ & $\begin{array}{l}\text { Coll. } \\
\text { date }\end{array}$ & $\delta \mathrm{C}^{14 \%} \%$ & $\delta \mathrm{C}^{13 \%} \%$ & $\Delta \%$ \\
\hline GU-169 & Jan. & $59.0 \pm 1.1$ & -23.3 & $58.4 \pm 1.1$ \\
GU-170 & April & $54.2 \pm 0.9$ & -24.8 & $54.2 \pm 1.0$ \\
GU-171 & July & $51.9 \pm 1.2$ & -21.6 & $50.9 \pm 1.3$ \\
GU-172 & Oct. & $63.2 \pm 1.0$ & -24.3 & $63.0 \pm 1.1$ \\
GU-173 & Dec. & $52.5 \pm 0.8$ & -23.6 & $52.1 \pm 0.9$
\end{tabular}

\section{Melbourne series}

Samples coll. by Meteorologic Office, in thermometer screen fitted with perspex hood for protection against dry deposition $\left(37^{\circ} 49^{\prime} \mathrm{S}\right.$ Lat, $144^{\circ} 58^{\prime}$ E Long).

Melbourne series, 1967

\begin{tabular}{llccc}
$\begin{array}{l}\text { Sample } \\
\text { no. }\end{array}$ & $\begin{array}{l}\text { Coll. } \\
\text { date }\end{array}$ & $\delta \mathrm{C}^{14} \%$ & $\delta \mathrm{C}^{13 \%} \%$ & $\Delta \%$ \\
\hline GU-174 & Jan. & $55.6 \pm 0.9$ & -25.0 & $55.6 \pm 1.0$ \\
GU-175 & April & $56.3 \pm 0.8$ & -26.7 & $56.9 \pm 0.9$ \\
GU-176 & July & $51.2 \pm 1.0$ & -20.1 & $49.8 \pm 1.0$ \\
GU-177 & Oct. & $52.3 \pm 0.9$ & -21.4 & $51.2 \pm 0.9$ \\
& & & & \\
Melbourne series, 1968 & & & \\
GU-178 & Jan. & $52.9 \pm 0.8$ & -19.1 & $51.1 \pm 0.9$ \\
GU-179 & April/May & $50.4 \pm 1.1$ & -20.6 & $49.0 \pm 1.2$ \\
GU-180 & July & $47.2 \pm 1.1$ & -21.7 & $46.3 \pm 1.2$ \\
GU-181 & Oct. & $49.6 \pm 1.1$ & -22.1 & $48.7 \pm 1.2$
\end{tabular}

Comment: $\mathrm{C}^{14}$ activities in Melbourne samples are generally low. The reason for this may be a "local" Suess effect, because there are some smokeproducing stacks within $1 \mathrm{mi}$ of sampling site and the harbour is ca. 3 mi away. 


\section{Stanley, Falkland Islands series}

Samples coll. outdoors by Meteorologic Office, Stanley, Falkland Is., in meteorologic thermometer screen ( $51^{\circ} 42^{\prime} \mathrm{S}$ Lat, $57^{\circ} 52^{\prime} \mathrm{W}$ Long).

\begin{tabular}{llccc}
$\begin{array}{l}\text { Stanley series, } \mathbf{1 9 6 8} \\
\begin{array}{c}\text { Sample } \\
\text { no. }\end{array}\end{array}$ & $\begin{array}{l}\text { Coll. } \\
\text { date }\end{array}$ & $\delta \mathrm{C}^{14} \%$ & $\delta \mathrm{C}^{13} \%$ & $\Delta \%$ \\
\hline GU-182 & Jan. & $55.1 \pm 1.0$ & -21.9 & $54.2 \pm 1.0$ \\
GU-183 & April & $56.3 \pm 0.8$ & -24.2 & $56.1 \pm 0.8$ \\
GU-184 & July & $52.4 \pm 0.8$ & -24.8 & $52.3 \pm 0.9$ \\
GU-185 & Oct. & $49.0 \pm 1.0$ & -24.7 & $49.0 \pm 1.0$
\end{tabular}

\section{Argentine Islands series}

Samples coll. by British Antarctic Survey in magnetic observatory (65 $15^{\prime} \mathrm{S}$ Lat, $64^{\circ} 16^{\prime} \mathrm{W}$ Long).

\section{Argentine Islands series, 1967}

\begin{tabular}{clccc}
$\begin{array}{c}\text { Sample } \\
\text { no. }\end{array}$ & $\begin{array}{c}\text { Coll. } \\
\text { date }\end{array}$ & $\delta \mathrm{C}^{14} \%$ & $\delta \mathrm{C}^{13 \%} \%$ & $\Delta \%$ \\
\hline GU-186 & April & $55.4 \pm 0.9$ & -20.4 & $54.0 \pm 1.0$ \\
GU-187 & July & $53.9 \pm 0.8$ & -20.9 & $52.6 \pm 0.9$ \\
GU-188 & Oct. & $54.7 \pm 0.9$ & -21.4 & $53.6 \pm 0.9$
\end{tabular}

\section{Argentine Islands series, 1968}

$\begin{array}{lllll}\text { GU-189 } & \text { April } & 53.2 \pm 0.9 & (-20.9) & 52.0 \pm 1.0 * \\ \text { GU-190 } & \text { Oct. } & 52.8 \pm 0.9 & (-20.9) & 51.5 \pm 0.9 *\end{array}$
Comment: * indicates that no mass spectrometric measurement was available for sample; a value of $-20.9 \pm 1 \%$ was assumed.

\section{Halley Bay series}

Samples coll. by British Antarctic Survey in magnetic hut during summer and in ozone hut during winter ( $75^{\circ} 31^{\prime} \mathrm{S} \mathrm{Lat,} 26^{\circ} 45^{\prime} \mathrm{W}$ Long).

\section{Halley Bay series, 1967}

\begin{tabular}{ccccc}
$\begin{array}{c}\text { Sample } \\
\text { no. }\end{array}$ & $\begin{array}{c}\text { Coll. } \\
\text { date }\end{array}$ & $\delta \mathrm{C}^{14} \%$ & $\delta \mathrm{C}^{13 \%} \%$ & $\Delta \%$ \\
\hline$G \mathrm{U}-191$ & Dec. & $55.8 \pm 0.9$ & -20.0 & $54.2 \pm 0.9$
\end{tabular}

\section{Halley Bay series, 1968}

$\begin{array}{lllll}\text { GU-192 } & \text { May } & 55.3 \pm 0.9 & -28.3 & 56.4 \pm 1.0 \\ \text { GU-193 } & \text { July } & 55.1 \pm 1.0 & -22.0 & 54.2 \pm 1.1 \\ \text { GU-194 } & \text { Oct. } & 52.2 \pm 1.0 & -22.9 & 51.6 \pm 1.1\end{array}$




\section{B. Upper atmospheric samples}

The following $\mathrm{C}^{14}$ activities were measured for $\mathrm{CO}_{2}$ coll. from the upper troposphere and lower stratosphere during the period June 1967 to December 1968.

Sampling was confined to flight paths within the region $50^{\circ}$ to $60^{\circ}$ $\mathrm{N} \mathrm{Lat}$, and $1^{\circ} \mathrm{E}$ to $8^{\circ} \mathrm{W}$ Long.

Atmospheric $\mathrm{CO}_{2}$ was adsorbed on $1 / 8$ in. pellets of molecular sieve, Linde Type 4A, using the techniques described by Godwin and Willis (Radiocarbon, 1964, v. 6, p. 134). Sampling time was $20 \mathrm{~min}$. and this proved sufficient for the collection of ca. $4.0 \mathrm{~L}-\mathrm{atm} . \mathrm{CO}_{2}$, using $2 \mathrm{~kg}$ sieve per sample. Adsorbed $\mathrm{CO}_{2}$ was recovered from the sieve material with steam displacement and coll. as $\mathrm{BaCO}_{3}$ by absorption in $\mathrm{Ba}(\mathrm{OH})_{2}$ / KOH solution (Harkness, 1970).

Upper atmospheric $\mathrm{CO}_{2}$

\begin{tabular}{|c|c|c|c|c|c|c|c|c|}
\hline $\begin{array}{c}\text { Sample } \\
\text { no. }\end{array}$ & & $\begin{array}{l}\text { Coll } \\
\text { date }\end{array}$ & & $\begin{array}{l}\text { Alt. } \\
(\mathrm{ft})\end{array}$ & $\begin{array}{l}\text { Tropo- } \\
\text { pause } \\
\text { ht. }(\mathrm{ft})\end{array}$ & $\delta \mathrm{C}^{14} \%$ & $\delta \mathrm{C}^{13 \%} \%$ & $\Delta \%$ \\
\hline 105 & 30 & June & 1967 & 41,000 & 39,000 & $87.7 \pm 1.4$ & -20.2 & $85.9 \pm 1.6$ \\
\hline GU-196 & 20 & Dec. & 1967 & 41,000 & 39,000 & $87.0 \pm 1.9$ & & $85.0 \pm 2.1$ \\
\hline GU-197 & 15 & Jan. & 1968 & 43,000 & 41,000 & $79.9 \pm 0.9$ & -18.3 & $77.5 \pm 1.0$ \\
\hline GU-198 & 15 & Feb. & 1968 & 39,000 & 35,000 & 74. & 17.8 & $72.0 \pm$ \\
\hline GU-199 & 15 & Feb. & 1968 & 31,000 & 35,000 & 58.3 & -17.1 & $55.8 \pm 0.7$ \\
\hline GU-200 & 15 & Mar. & 1968 & 43,000 & 41,000 & 62.8 & -20.5 & $61.4 \pm 2.1$ \\
\hline GU-201 & 19 & Mar. & 1968 & 31,000 & 28,000 & $62.8 \pm$ & -19.8 & $61.1 \pm 0.9$ \\
\hline GU-202 & 19 & Mar. & 1968 & 25,000 & 28,000 & 64.6 & -20.3 & $63.0 \pm 1.0$ \\
\hline GU-203 & 26 & Mar. & 1968 & 41,000 & 38 , & 72.3 & -20.2 & $70.6 \pm 0.8$ \\
\hline & 26 & Apr. & 1968 & 41,000 & 39,000 & $73.6 \pm 0.9$ & -16.9 & $70.7 \pm 0.7$ \\
\hline GU-2C & 30 & Apr. & 1968 & 39,000 & 29,000 & $63.7 \pm$ & -22.4 & $62.8 \pm 2.2$ \\
\hline GU-206 & 30 & Apr. & 1968 & 27,000 & 29,000 & $62.1 \pm 0.8$ & -19.7 & $60.4 \pm 0.9$ \\
\hline GU-207 & 21 & May & 1968 & 39,000 & 34,000 & $79.5 \pm 1.6$ & -21.8 & $78.3 \pm 1.6$ \\
\hline GU-208 & 4 & Nov. & 1968 & 45,000 & 41,000 & $74.8 \pm 0.8$ & -18.9 & $72.7 \pm 0.9$ \\
\hline GU-209 & 6 & Dec. & 1968 & 41,000 & 39,000 & $72.5 \pm 0.6$ & -20.3 & $70.8 \pm 0.7$ \\
\hline
\end{tabular}

III. BLOOD PROTEIN SAMPLES

Data reported here are derived from the protein fraction separated from human blood plasma collected in S Scotland. Each sample represents a composite prepared from the whole blood of 10 donors. Collection date quoted is accurate to within \pm 5 days. 
Blood protein, S Scotland

\begin{tabular}{|c|c|c|c|c|c|c|}
\hline \multirow{2}{*}{$\begin{array}{l}\text { Sample } \\
\text { no. } \\
\text { GU-210 }\end{array}$} & \multicolumn{3}{|c|}{$\begin{array}{c}\text { Sample } \\
\text { date }\end{array}$} & \multirow{2}{*}{$\begin{array}{c}\delta \mathrm{C}^{14} \% \\
-3.6 \pm 0.5\end{array}$} & \multirow{2}{*}{$\begin{array}{r}\delta \mathrm{C}^{13 \%} \% \\
-28.4\end{array}$} & \multirow{2}{*}{$\frac{\Delta \%}{-2.9 \pm 0 .}$} \\
\hline & 26 & Oct. & 1952 & & & \\
\hline GU-211 & 20 & Sept. & 1953 & $-8.1 \pm 0.6$ & -26.4 & $-7.9 \pm 0.6$ \\
\hline GU-212 & 1 & Apr. & 1954 & $-5.4 \pm 0.7$ & -26.2 & $-5.1 \pm 0.8$ \\
\hline GU-213 & 23 & Mar. & 1955 & $-1.6 \pm 0.5$ & -26.2 & $-1.3 \pm 0.5$ \\
\hline GU-214 & 5 & May & 1956 & $-7.8 \pm 0.6$ & -29.5 & $-7.0 \pm 0.7$ \\
\hline GU-215 & 26 & Sept. & 1957 & $-5.0 \pm 0.7$ & -30.8 & $-3.9 \pm 0.8$ \\
\hline GU-216 & 11 & Feb. & 1960 & $9.1 \pm 0.6$ & -26.5 & $9.4 \pm 0.7$ \\
\hline GU-217 & 23 & May & 1961 & $16.4 \pm 0.9$ & -27.2 & $16.9 \pm 1.0$ \\
\hline GU-218 & 7 & Apr. & 1962 & $9.9 \pm 0.9$ & -29.4 & $10.8 \pm 0.9$ \\
\hline GU-219 & 15 & July & 1963 & $32.0 \pm 1.0$ & -32.3 & $33.9 \pm 1.1$ \\
\hline GU-220 & 9 & Feb. & 1964 & $44.4 \pm 1.0$ & -29.4 & $45.6 \pm 1.0$ \\
\hline GU-221 & 5 & Mar. & 1965 & $60.1 \pm 0.8$ & -29.5 & $61.5 \pm 0.8$ \\
\hline GU-222 & 17 & Oct. & 1966 & $65.4 \pm 0.8$ & -27.2 & $66.2 \pm 0.8$ \\
\hline GU-223 & 15 & Nov. & 1966 & $64.0 \pm 0.7$ & -30.0 & $65.6 \pm 0.8$ \\
\hline GU-224 & 30 & Dec. & 1966 & $64.5 \pm 0.7$ & -27.9 & $65.4 \pm 0.7$ \\
\hline GU-225 & 8 & Apr. & 1967 & $64.2 \pm 0.6$ & -28.4 & $65.3 \pm 0.7$ \\
\hline GU-226 & 27 & Oct. & 1967 & $62.7 \pm 0.7$ & -33.2 & $65.3 \pm 0.8$ \\
\hline GU-227 & 10 & July & 1968 & $63.2 \pm 1.0$ & -26.3 & $63.4 \pm 1.0$ \\
\hline
\end{tabular}

Comment: $\mathrm{C}^{14}$ concentrations in blood protein indicate significant correlation with modifying influences on atmospheric $\mathbf{C}^{14}$ levels, viz. Suess effect and bomb effect. Blood protein $\mathrm{C}^{14}$ levels, however, did not reach peak concentrations attained in atmosphere, reflecting variations in source of carbon in diet and possibly tissue "turnover" time (Harkness and Walton, 1969).

\section{REFERENCES}

Baxter, M. S., Ergin, M., and Walton, A., 1969, Glasgow University radiocarbon measurements I: Radiocarbon, v. 11, p. 43-52.

Broecker, W. S. and Olson, E. A., 1961, Lamont radiocarbon measurements VIII: Radiocarbon, v. 3, p. 176-204.

Godwin, H. and Willis, E. H., 1964, Cambridge University natural radiocarbon measurements VI: Radiocarbon, v. 6, p. 116-137.

Harkness, D. D., 1970, Artificial carbon-14 in the atmosphere and biosphere: Ph.D. thesis, Univ. of Glasgow.

Walton, A., Ergin, M., and Harkness, D. D., 1969, Carbon-14 concentrations in the atmosphere and carbon dioxide exchange rates: Paper, C.A.C.R. symposium on atmospheric trace constituents and atmospheric circulation, Heidelberg, Germany, Sept. 8-13, 1969 (Jour. Geophys Research, 1970, in press). 